\title{
Trends in the treatment of pancreatic cancer in Japan
}

\author{
Ryota Matsuki, Takaaki Arai, Masaharu Kogure, Yutaka Suzuki, Yoshihiro Sakamoto*
}

Department of Hepato-Biliary-Pancreatic Surgery, Kyorin University Hospital, Mitaka, Tokyo, Japan.

SUMMARY Pancreatic cancer is known to have the poorest prognosis among digestive cancers. With the development of new chemotherapeutic agents and introduction of multidisciplinary therapy, however, the treatment outcomes for pancreatic cancer have dramatically improved over the past two decades. The keys to successful treatment will be accurate assessment of resectability [resectable (R), borderline resectable (BR) or unresectable (UR)] at the time of diagnosis and prompt adoption of an appropriate multidisciplinary treatment strategy. Prep-02/JSAP-05 trial which is an RCT of upfront surgery versus neoadjuvant chemotherapy using GEM and S-1 (GS) and subsequent surgery for R-PDAC in Japan indicated neoadjuvant chemotherapy had a longer overall survival (OS) than those undergoing upfront surgery (36.7M vs. 26.6M, $p=0.015)$. In a retrospective multicenter study in Japan reported that in BR-PDAC, median survival time (MST) in the pretreatment group was significantly better than that in the upfront surgery group (25.7 months $v s .19 .0$ months, $p=0.015)$ according to a propensity score matching analysis. Another retrospective multicenter study with UR-LA PDAC in Japan reported that conversion surgery was more beneficial for patients with more than 8 months of preoperative therapy than those with less than 8 months of that therapy. Various clinical trials on pancreatic cancer are ongoing, and the results of trials on chemotherapeutic regimens and multidisciplinary treatments will be of further interest.

Keywords pancreatic cancer, resectability, multidisciplinary treatment

\section{Introduction}

Pancreatic cancer is known to have the poorest prognosis among digestive cancers; only $15-20 \%$ of cases are resectable while $30-40 \%$ of cases involve locally advanced cancer and $50-60 \%$ of cases involve distant metastatic cancer, which is unresectable (1). With the development of new chemotherapeutic agents and introduction of multidisciplinary therapy, however, the treatment outcomes for pancreatic cancer have dramatically improved over the past two decades. The treatment strategy for pancreatic cancer depends on the resectability of each cancer. The resectability of pancreatic cancer was first classified in the NCCN guidelines in 2004, and further objective classification based on anatomy and tumor extension on CT images was proposed by the M. D. Anderson Cancer Center (MDACC) in 2006 (2). In Japan, in 2016, the 7th Edition of the Classification of Pancreatic Cancer clearly stated that the resectability of pancreatic cancer should be classified as resectable (R), borderline resectable (BR), or unresectable (UR) based on local extension and the presence or absence of distant metastasis (3). The keys to successful treatment will be accurate assessment of resectability at the time of diagnosis and prompt adoption of an appropriate multidisciplinary treatment strategy

\section{Multidisciplinary treatment for pancreatic ductal adenocarcinoma (PDAC)}

\subsection{Adjuvant treatment for PDAC}

Since the beginning of the 21 st century, gemcitabine (GEM) has frequently been used to treat UR pancreatic cancer. In response to that trend, the CONKO-001 trial in Germany (4) and the JSAP-02 trial in Japan (5) were conducted as randomized controlled trials (RCTs) on adjuvant therapy after surgery for R-PDAC. The results of these trials indicated that patients who received GEM for 3-6 months after surgery for R-PDAC had a longer recurrence-free survival (RFS) than the observation group. Subsequently, the JASPC 01 study in Japan (6) indicated that the administration of adjuvant S-1 significantly improved not only RFS but also overall survival (OS) in comparison to administration of GEM, and this finding was announced at the American Gastrointestinal Cancer Symposium (ASCO-GI) in 2013. Since then, administration of S-1 as adjuvant 
Table 1. Results of perioperative adjuvant chemotherapy for resectable pancreatic cancer

\begin{tabular}{|c|c|c|c|c|c|c|}
\hline Study & Country & Year & Journal & Therapy & $n$ & $\operatorname{MST}(\mathrm{M})$ \\
\hline CONKO-001 & Germany & 2007 & JMA & $\begin{array}{l}\text { GEM 6M } \\
\text { observation }\end{array}$ & $\begin{array}{l}179 \\
175\end{array}$ & $\begin{array}{l}\text { 13.4 PFS } \\
6.9 \text { PFS }\end{array}$ \\
\hline JSAP-02 & Japan & 2009 & Br J Cancer & $\begin{array}{l}\text { GEM 3M } \\
\text { observation }\end{array}$ & $\begin{array}{l}58 \\
60\end{array}$ & $\begin{array}{l}\text { 11.4 PFS } \\
\text { 5.0 PFS }\end{array}$ \\
\hline JASPAC01 & Japan & 2016 & Lancet & $\begin{array}{l}\text { S-1 6M } \\
\text { GEM 6M }\end{array}$ & $\begin{array}{l}187 \\
190\end{array}$ & $\begin{array}{l}46.5 \\
25.5\end{array}$ \\
\hline ESPAC-4 & European study group & 2017 & Lancet & $\begin{array}{l}\text { GEM+Cape 6M } \\
\text { GEM 6M }\end{array}$ & $\begin{array}{l}364 \\
366\end{array}$ & $\begin{array}{l}28.0 \\
25.5\end{array}$ \\
\hline PRODIGE24-ACCORD24/CCTG PA6 & Canada & 2018 & N Engl J Med & $\begin{array}{l}\text { mFOLFIRINOX } 6 \mathrm{M} \\
\text { GEM 6M }\end{array}$ & $\begin{array}{l}247 \\
246\end{array}$ & $\begin{array}{l}54.4 \\
35.0\end{array}$ \\
\hline Prep-02/JSAP-05 & Japan & 2019 & $\mathrm{~J}$ Clin Oncol & $\begin{array}{l}\text { Neo GEM+S-1 2M } \\
\text { Upfront surgery }\end{array}$ & $\begin{array}{l}182 \\
180\end{array}$ & $\begin{array}{l}36.7 \mathrm{OS} \\
26.6 \mathrm{OS}\end{array}$ \\
\hline
\end{tabular}

GEM: gemcitabine; Neo: neoadjuvant therapy; MST: median survival time; PFS: progression free survival.

chemotherapy has become the standard treatment for R-PDAC in Japan (Table 1). In addition, since 2016, two clinical trials of adjuvant chemotherapy [ESPAC-4: GEM vs. GEM plus capecitabine](7) and [PRODIGE24-ACCORD24/CCTG PA6 trial: GEM vs. MFOLFIRINOX](8) yielded positive results. Based on the results of these phase III trials, the2019 Clinical Practice Guidelines for Pancreatic Cancer (9) proposed adding the use of GEM plus capecitabine and modified FOLFIRINOX as recommended adjuvant chemotherapies. However, there are no data on the use of either of these adjuvant agents in Japan, and they are not recommended by Japanese guidelines.

\subsection{Neoadjuvant therapy for PDAC}

\subsubsection{Neoadjuvant therapy for resectable PDAC}

Prep-02/JSAP-05 trial (10) was conducted to clarify the significance of neoadjuvant chemotherapy for R-PDAC. This trial was an RCT of upfront surgery versus neoadjuvant chemotherapy using GEM and S-1 (GS) and subsequent surgery for R-PDAC. Both groups received adjuvant chemotherapy with $\mathrm{S}-1$. The results of this study, announced at the ASCO-GI in 2019, were that patients receiving neoadjuvant chemotherapy had a longer OS than those undergoing upfront surgery. Based on these results, neoadjuvant GS and adjuvant S-1 therapy became the standard treatment for R-PDAC in Japan after 2019.

\subsubsection{Neoadjuvant therapy for BR-PDAC}

In Japan, the 2019 Clinical Practice Guidelines for Pancreatic Cancer proposed surgical resection for BR-PDAC after neoadjuvant therapy followed by reevaluation of treatment efficacy and resectability. Upfront surgery for BR-PDAC is not recommended. Although several RCTs on neoadjuvant therapy for BRPDAC are being conducted in Europe, the United States, and Japan, RCTs have yet to provide evidence of the efficacy of neoadjuvant therapy.

In a retrospective multicenter study in Japan, Nagakawa et al. reported that among 884 patients with BR-PDAC (530 patients in the pretreatment group and 354 in the upfront surgery group), median survival time (MST) in the pretreatment group $(n=297)$ was significantly better than that in the upfront surgery group $(n=297)(25.7$ months vs. 19.0 months, $p=0.015)$ according to a propensity score matching analysis (11).

\subsection{Chemotherapy for UR-PDAC}

The use of GEM as the standard treatment for UR-PDAC lasted for more than a decade starting in 2000. However, since FOLFIRINOX therapy was approved in 2013 and the combination of GEM and nab-paclitaxel was approved in 2014, these two combination therapies have been widely used in Japan. The 2019 Clinical Practice Guidelines for Pancreatic Cancer also recommend the use of GEM monotherapy, S-1 monotherapy, and the combination of GEM and erlotinib in addition to the two aforementioned therapies. New anti-cancer therapies using molecularly targeted drugs and immune checkpoint inhibitors are expected in the near future.

\subsection{Conversion surgery}

UR-PDAC can be classified into UR-locally advanced (UR-LA) and UR-metastatic cancer. The 2019 Clinical Practice Guidelines for Pancreatic Cancer suggest that conversion surgery after multidisciplinary treatment could be a treatment option for UR-LA PDAC because favorable OS and/or RFS can be expected. In a retrospective multicenter study involving 97 patients with UR-LA PDAC in Japan, conversion surgery was more beneficial for patients with more than 8 months of preoperative therapy than those with less than 8 months of that therapy (12). Conversion surgery should be performed after tumor markers such as CA19-9 have decreased sufficiently. Although conversion surgery is expected to prolong survival for patients if it is 
performed safely, it should be performed carefully by a specialized facility because it requires highly technical skills, such as combined resection and reconstruction of the hepatic artery and portal vein.

\section{Conclusion}

The keys to successful treatment of pancreatic cancer will be accurate assessment of resectability and adoption of an appropriate multidisciplinary treatment strategy. Ongoing clinical trials on pancreatic cancer will lead the way to new chemotherapeutic regimens and multidisciplinary treatments.

Funding: None.

Conflict of Interest: The authors have no conflicts of interest to disclose.

\section{References}

1. Gillen S, Schuster T, Meyer Zum Büschenfelde C, Friess H, Kleeff J. Preoperative/neoadjuvant therapy in pancreatic cancer: a systematic review and meta-analysis of response and resection percentages. PLoS Med. 2010; 7:e1000267.

2. Varadhachary GR, Tamm EP, Abbruzzese JL, Xiong HQ, Crane CH, Wang H, Lee JE, Pisters PW, Evans DB, Wolff RA. Borderline resectable pancreatic cancer: definitions, management, and role of preoperative therapy. Ann Surg Oncol. 2006; 13:1035-1046.

3. Japan Pancreas Society. Classification of Pancreatic Carcinoma, 7th Edition. 2016; Kanehara, Tokyo, Japan. (in Japanese)

4. Oettle H, Post S, Neuhaus P, et al. Adjuvant chemotherapy with gemcitabine $v s$ observation in patients undergoing curative-intent resection of pancreatic cancer. A randomized controlled trial. JAMA. 2007; 297:267-277.

5. Ueno H, Kosuge T, Matsuyama Y, Yamamoto J, Nakao A, Egawa S, Doi R, Monden M, Hatori T, Tanaka M, Shimada M, Kanemitsu K. A randomized phase III trial comparing gemcitabine with surgery-only in patients with resected pancreatic cancer: Japanese Study Group of Adjuvant Therapy for Pancreatic Cancer. Br J Cancer. 2009; 101:908-915.
6. Uesaka K, Boku N, Fukutomi A, et al. Adjuvant chemotherapy of S-1 versus gemcitabine for selected pancreatic cancer: A phase 3, open-label, randomized, noninferiority trial (JASPAC 01). Lancet. 2016; 388:248-257.

7. Neoptolemos JP, Palmer DH, Ghaneh P, et al. Comparison of adjuvant gemcitabine and capecitabine with gemcitabine monotherapy in patients with resected pancreatic cancer (ESPAC-4): A multicentre, open-label, randomised, phase 3 trial. Lancet. 2017; 389:1011-1024.

8. Conroy T, Hammel P, Hebbar M. et al. FOLFIRINOX or gemcitabine as adjuvant therapy for pancreatic cancer. N Engl J Med. 2018; 379:2395-2406.

9. Japan Pancreas Society. The 2019 Clinical Practice Guidelines for Pancreatic Cancer. http://www.suizou.org/ pdf/pancreatic_cancer_cpg-2019.pdf (accessed March 11, 2021). (in Japanese)

10. Unno M, Motoi F, Matsuyama Y, Satoi S, Matsumoto I, Aosasa S, Shirakawa H, Wada K, Fujii T, Yoshitomi H, TakahashiS, Sho M, Ueno H, Kosuge T. Randomized phase II/III trial of neoadjuvant chemotherapy with gemcitabine and S-1 versus upfront surgery for resectable pancreatic cancer (Prep-02/JSAP-05). J Clin Oncol. 2019; 37: 4 suppl 189.

11. Nagakawa Y, Sahara Y, Hosokawa Y et al. Clinical impact of neoadjuvant chemotherapy and chemoradiotherapy in borderline resectable pancreatic cancer: Analysis of 884 patients at facilities specializing in pancreatic surgery. Ann Surg Oncol. 2019; 26:1629-1636.

12. Satoi S, Yamaue H, Kato K et al. Role of adjuvant surgery for patients with initially unresectable pancreatic cancer with a long-term favorable response to non-surgical anticancer treatments: Results of a project study for pancreatic surgery by the Japanese Society of Hepato-BiliaryPancreatic Surgery. J Hepatobiliary Pancreat Sci. 2013; 20:590-600.

Received March 16, 2021; Revised March 22, 2021; Accepted March 24, 2021.

*Address correspondence to:

Yoshihiro Sakamoto, Department of Hepato-Biliary-Pancreatic Surgery, Kyorin University Hospital, 6-20-2 Shinkawa, Mitaka, Tokyo 181-8611, Japan.

E-mail: yosakamo@ks.kyorin-u.ac.jp

Released online in J-STAGE as advance publication March 26, 2021. 